\title{
A design of SMS alarm system based on 51 SCM and GPS positioning module
}

\author{
Xiaowei Chen ${ }^{1, a}$, Wenyu Niu ${ }^{2, b}$, Jin $\mathrm{Xu}^{2, \mathrm{c}}$ \\ ${ }^{1}$ Wuhan University of Technology, Wuhan 430000, China \\ ${ }^{2}$ Wuhan University of Technology, Wuhan 430000, China \\ a396502308@qq.com, b229544498@qq.com, '2570160367@qq.com
}

Keywords: MCS-51 SCM, GPS, GSM SMS module.

\begin{abstract}
A design of alarm system basing on 51 SCM used dedicated GPS positioning module and GSM SMS module, which owns higher positioning accuracy and lower signal requirement, comparing with ordinary mobile phone. It can send location information to the outside world for help accurately and timely, reduce unnecessary casualties to maximum extent and have broad application prospects and good feasibility.
\end{abstract}

\section{Introduction}

The aim of SMS alarm system basing on 51 SCM and GPS positioning module is to realize to alarm location fast and reduce unnecessary casualties to maximum extent. At present, the development of smart phones is rapid, but its main function is focus on communication, Internet and the GPS positioning function of its own is not as good as dedicated GPS positioning module naturally. So, it is very important to send location information to the outside world for help accurately and timely when the accident occurred.

\section{The overall design of the system}

The alarm system consists of four parts, 51 microcontroller, buttons, LEA-5S GPS module, power module and GSM SMS module. The design principle is as follows. Power module is divided into two parts, and one part is used to provide 5V/2A power supply for the GSM module, the other is used to provide $5 \mathrm{~V}$ power supply for the microcontroller; GPS module obtains data from satellites in real time and transfer it to the microcontroller via TXD and RXD serial ports of communication; SMS module obtains information from the microcontroller serial ports and send location information to the outside world for help; Button play the role of triggering microcontroller to receive GPS module data and control messages module. Alarm system hardware block diagram is shown in Fig. 1.1.

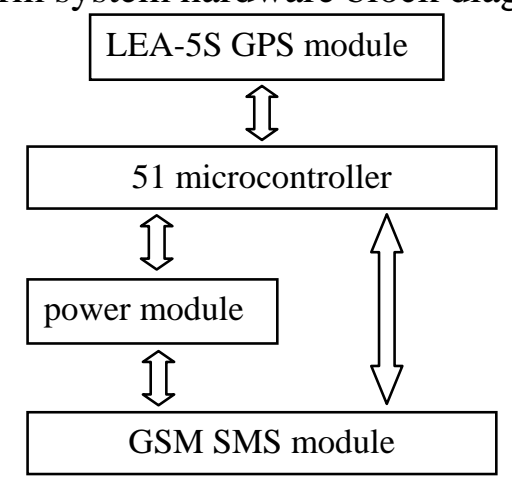

Fig 1.1 Alarm system hardware structures

Workflow of alarm system is as follows. Start switch, GPS module, GSM SMS modules' initialized waiting, and it can begin to work if it is normal, if not, please restart module; Then detect whether the button is triggered, and if trigger is detected, microcontroller receive information from the data of GPS, which conform specific rules through RXD serial port, recognize the data according to the rules and then future deal with the identified information to extract the required information; 
Finally, it will send a distress message to the specified users through controlling GSM model by microcontroller, which include the latitude and longitude of the location. The workflow is shown in Fig. 1.2.

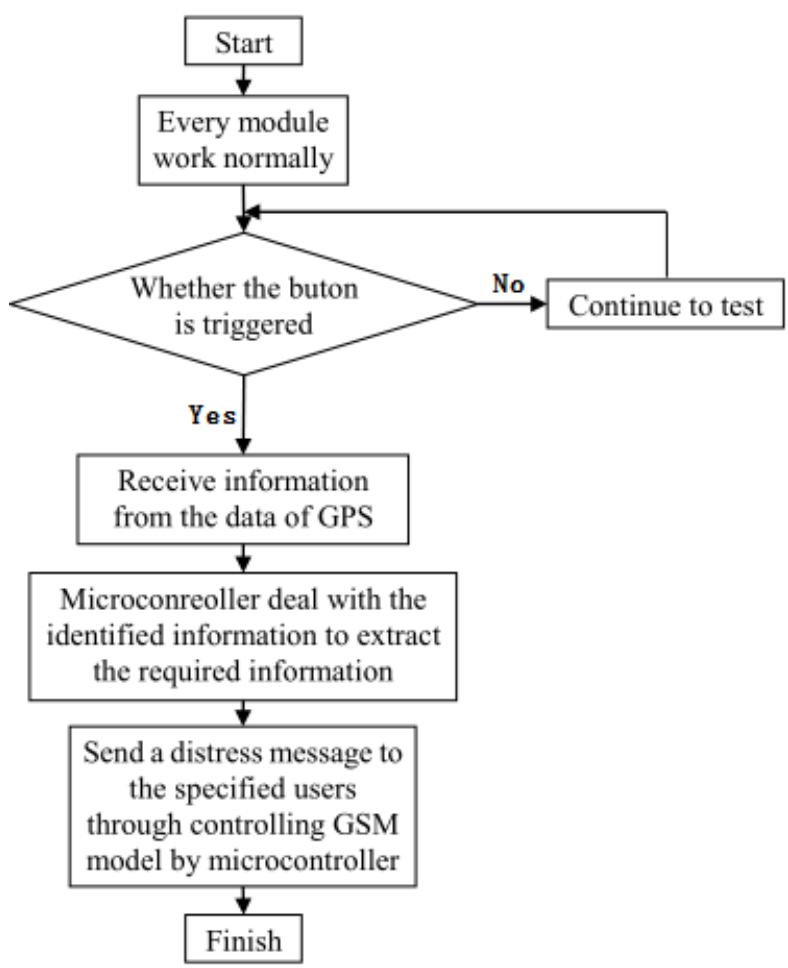

Fig. 1.2 Work flow chart of alarm system

\section{Hardware Design}

Each hardware module's parameter selection. The system's control unit is STC89C52 microcontroller, whose program memory space is $8 \mathrm{~K}$ bytes, data storage space is 512 bytes, power provided is $5 \mathrm{~V}$ supply, and $\mathrm{P} 1$ is the quasi-bidirectional/weak pull, therefore it does not need to add an external pull-up resistor to do the function as I/O port, besides, TXD and RXD are serial communication port. As for Loudspeaker controlling, it uses switching action of Q28550 transistor model. GSM SMS module uses GTM900B, and its main feature is its power supply is voltage of 5V, the current supply is 1A, 2A peak current as communication. More importantly, it supports the MCU, USB transferring TTL directed connection such as computer serial port (232 level) connection and microcontroller. Its parameters introduced are shown in Table 1.1. GPS positioning module use LEA-5S-type development board, equipped with a high-performance 50-channel U-blox5 technology, and have more than one million effective correlates 32-channel acquisition engine processing capability to making the module be massively parallel search. Its main characteristics are shown in Table 1.1.

Overall circuit design system. MCU clock circuit uses HC49/S-HC/49SS passive crystal, which connects interface XTAL1 and XTAL2 with other two 30pF ceramic capacitors and its nominal frequency is 11.0592MHZ; Reset circuit using buttons and electrolytic capacitors series connection; Controlling of loudspeaker uses the switching function of transistors Q28550, and the base is connected to P1.5 port, emitter connects to the positive of loudspeaker, and collector connects to $5 \mathrm{~V}$ power supply. SMS GPS module has three interfaces: TXD, RXD, VCC, GND., and the system only need to use VCC, GND and TXD, which is used to send data to the microcontroller. GSM SMS interface module has VCC, GND, TXD, RST and RI ports and VCC, GND, RXD are used. SMS module's power requirements are high, and need 1-2A current to work normally. Overall system circuit diagram is shown in Fig. 1.3. 
Table 1.1 GSM module parameters introduction

\begin{tabular}{|c|c|}
\hline Items & description \\
\hline product name & SR_GTM900B GPRS module \\
\hline major function & Voice communication, sending and receiving of SMS messages, GPRS \\
data transmission
\end{tabular}

Table 1.2 GPS module main performance characteristics

GPS receiver with high precision and high-performance

Dc power input with 5V, Dc power output with 3.3V,

Antenna opening, Short circuit detection function

UART port

Level of a serial port with RS232 and USB2.0

Convenient to connect the computer with GPS test software

Power supply and working turntable indicator light

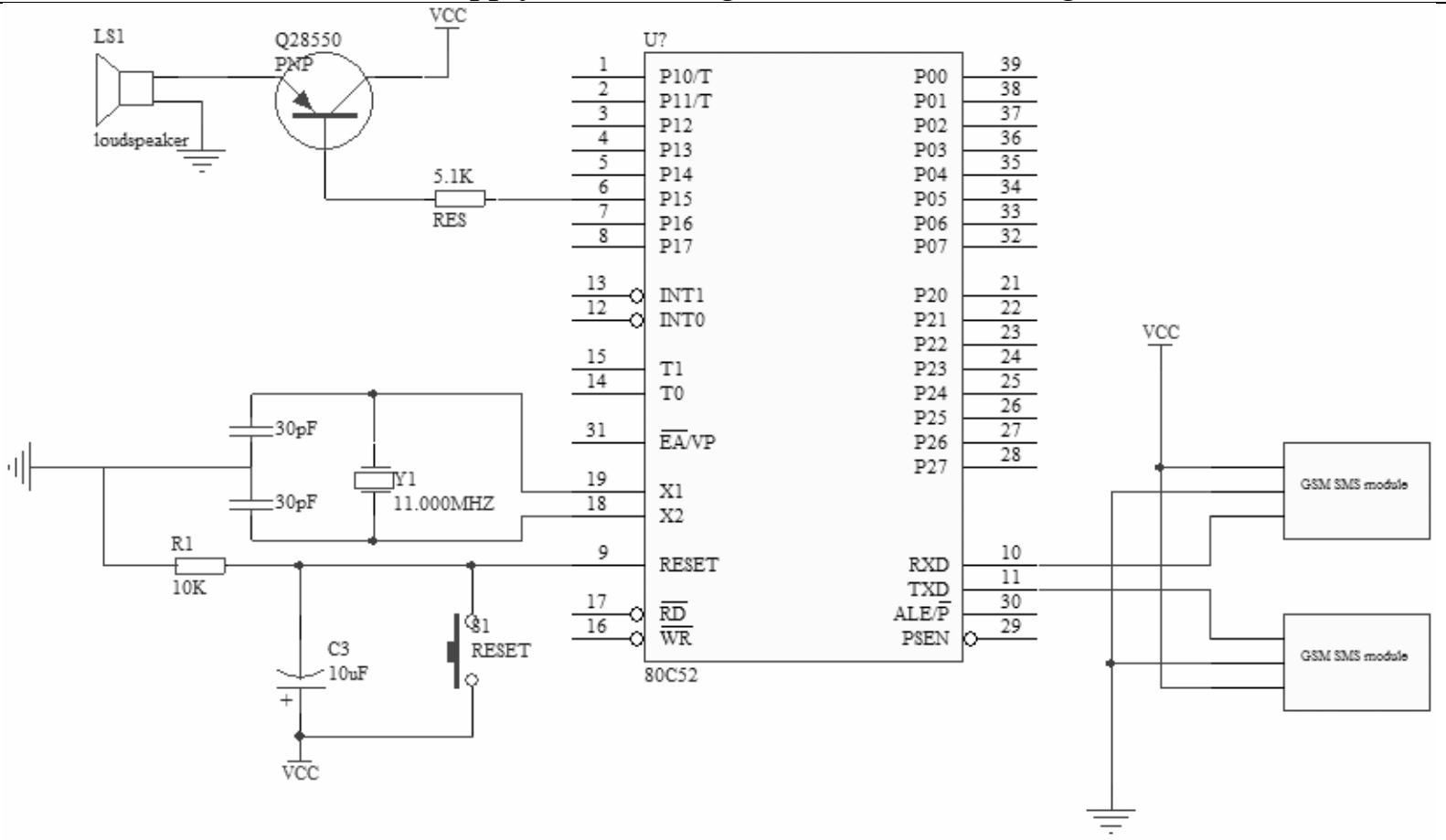

Fig. 1.3 Overall system schematic

\section{Software Design}

System program consists of two parts, and the one is extraction of GPS data, the other is Coded information of SMS message. It will elaborate program designing ideas from above two aspects. GPS positioning module collect the information, including location warning, positioning quality, location status, elevation, satellite, speed, latitude and longitude, and the system mainly collect latitude and longitude to achieve spatial orientation. Transmitting information of GPS module is a total of 5 sections, including \$GPGGA, \$GPRMC, \$GPVTG, \$GPGSA and \$GPGSV, and the \$GPGGA and \$ GPRMC sections contain longitude and latitude information. System select \$ GPGGA as longitude and latitude data extraction section, and the principle is that it firstly determine the $\$$ start code, then 
judge G, P and G these three yards, and finally judge the fifth code, and if it is G, it is \$GPGGA and determination of pieces of information is all finished. Then it only needs to extract several letters of data after $\mathrm{N}$ and $\mathrm{E}$, representing longitude and latitude, and data extraction process is completed.

Several major commands of controlling GSM module to send SMS text messages by SCM are as follows. $\mathrm{A}, \mathrm{AT}+\mathrm{CMGF}=0$ and $\mathrm{AT}+\mathrm{CMGS}=*$, the most important of it is to determine representation digital of "*", and coding of SMS module which send Chinese characters controlled by SCM is the PDU code. The following describes the code of system sending distress content.

Remove the "+" sign of center number of the message, and determine whether the length is an even number, if not, add $\mathrm{F}$ in the end, for example, "8613800546500F", then, do the same processing for the set of phone number. Part processing of SMS is to convert a string to Unicode code, for example, "In danger, please timely rescue, longitude: **, latitude: **", and the Unicode code is "9047523053719669FF0C8BF753CA65F662A26551FF0C7ECF5EA64E3AFF1A002A002AFF0C 7 EF45EA64E3AFF1A002A002A", and then divide the length of this code is by 2, retain two hexadecimal numbers, referred to as $n$, assemble the resulting number again with code, referred to as msg, and finally, assemble several converted code. Add the string "11000D91" before the phone number, add 0008A7 after the phone number, finally add msg, the whole string of code referred to as all, then all is done.

At first, SCM send "AT" to the SMS module through a serial port TXD, return "OK", then send "AT + CMGS = n", finally send "all" code string, send 1A with hexadecimal, and SMS is end.

\section{Prospects and Conclusions}

Alarm system basing on 51 SCM used dedicated GPS positioning module and GSM SMS module, which owns higher positioning accuracy and lower signal requirement. It can send location information to the outside world for help accurately and timely, reduce unnecessary casualties to maximum extent and have broad application prospects and good feasibility.

\section{References}

[1] Guzman-Lopez J, Buisson Y, Strutton P H, Bronstein A M. Interaction between vestibulo-spinal and corticospinal systems: a combined caloric and transcranial magnetic stimulation study.[J]. Experimental Brain Research, 2011, 2141: 1-20.

[2] Bettez Neil D, Groffman Peter M. Denitrification potential in stormwater control structures and natural riparian zones in an urban landscape. [J]. Environmental Science \&amp; Technology, 2012, 4620: 5-30.

[3] Henri C. Dekker, Junya Sakaguchi, Takaharu Kawai. Beyond the contract: managing risk in supply chain relations [J]. Management Accounting Research, 2013, 6-20.

[4] Jung Yi Lin, J. H. Sossa, H. Xu. Using Evolutionary Computation on GPS Position Correction [J]. The Scientific World Journal, 2014, 2014: 5-18.

[5] Callan, James. Multilateration: Radar Is Out [J]. Avionics Magazine, 2014, 382: 7-23.

[6] Zengke Li, Jian Wang, Binghao Li, Jingxiang Gao, Xinglong Tan. GPS/INS Odometer Integrated System Using Fuzzy Neural Network for Land Vehicle Navigation Applications [J]. Journal of Navigation, 2014, 676: 7-34. 